\title{
A preliminary optimization study of a SWATH-USV
}

\author{
Baojiang Wang, Yan Shi, Songling Yang, Shasha Gao, Zhiwei Cao
}

\begin{abstract}
In this paper, we designed a kind of Swath-USV through corresponding ship hull parameters selected as design variables to identify the USV performance (Buoyancy, Stability, Rapidity, Maneuverability, And Sea-Keeping) as the comprehensive optimization objective function. First, we selected the range of design variables and constraint conditions, established the comprehensive optimization mathematical model of the ship hull and constructed the optimization methods. Then, we applied a multi-objective optimization design and analysis software for optimal comprehensive performance and took place a set of comprehensive optimization calculations. In the calculation process, Genetic algorithm was selected as the core algorithm to record the best five individual information, and then the second calculation was conducted in combination with itself and other algorithms. By comparing the fitness function values of the hybrid algorithms of discrete genetic iterations, it was found that the genetic plus chaos algorithm of 4000 generations has strong robustness, high optimization ability and less short running time. The second is genetic plus compound algorithm. Finally, the optimal ship hull parameters for the integrated performance of small waterline catamaran were generated. The research results can provide references for the comprehensive optimization of multi-objective, multi-variable and multi-constraint conditions of the performance optimization of small waterline catamaran.
\end{abstract}

Index Terms- Swath-USV; Multi-disciplinary comprehensive optimization; Mathematical model; Genetic algorithm; Hybrid algorithm

\section{INTRODUCTION}

Small Waterline Catamaran (SWATH-USV), as a high-performance ship, has become a hot ship in recent years because of its wide deck area, good seakeeping and low resistance at high speed [1] and using the optimization technology of the unmanned craft, we can see that the boat type is also gradually hot. Currently, SHIPFLOW and NASTRAN commercial computing software [2] were utilized to demonstrate and study the ship types. Currently, SHIPFLOW and NASTRAN commercial computing software are used to demonstrate and study ship type. Chen Xiaona [3] established the mathematical model of the catamaran, chooses the corresponding design variables and establishes the optimization scheme. Finally, through the analysis of the optimization results, preliminary optimization of the ship form is generated. But in the optimization scheme, the influence of the head shape changement and appendage part on the resistance performance is not considered. Xu Jie [4] applied multidisciplinary design optimization [5] to the design of SWATH-USV to improve hull design. Based on the hull resistance performance, Deng Fang and Deng Weibin [6] optimized the catamaran design, and concluded that the

Baojiang Wang, Yan Shi, Songling Yang, Shasha Gao, Zhiwei Cao, School of Naval Architecture \& Ocean Engineering, Jiangsu University of Science and Technology, Zhenjiang, Jiangsu, China ) wave-making resistance is inversely proportional to the center distance of the hull and the residual resistance is inversely proportional to the length-width ratio range of the hull.

The traditional single objective optimization design is only to optimize a certain performance of the ship, so it ignores the interaction between multiple disciplines, so it cannot achieve the best design scheme. At the same time, ship design will always have constraints on each other' s performance, such as the reconnaissance warship between the rapidity and stealth of the trade-off, which involves the design process as a designer how to distinguish and how to distribute the weight relationship between the performance. At this time, the application of multidisciplinary design theory [7] will become very necessary. In the optimization design, in addition to establishing the corresponding mathematical model, the selection and improvement of the algorithm have an important impact on the realization of the best design scheme.

\section{OPTIMIZATION MODEL OF SWATH-USV}

Considering the factors influencing the performance of the small waterline catamaran, the comprehensive performance including rapidity, maneuverability and Seakeeping source arrangement was studied in this paper, and a comprehensive optimization mathematical model of navigation performance was established.

\section{A . Variable design variables for ship performance}

\section{a. Rapidity design variables}

Usually, ship resistance and propulsion efficiency were used to characterize ship rapidity. Therefore, from these two aspects, we selected rapidity design variables. Design variables for resistance:Displacement,Block coefficient,Speed,Ratio of length to diameter of a floating body,Prop length,Hydrofoil parameters;Design variables for propulsion:Propeller diameter,Speed,Pitch ratio.Comprehensive consideration:Length of submersible body,Dive Diameter ( $D_{1}$ ),Prop Length ( $L_{s}$ ),Maximum Width Of Pillar ( $\left.t_{s}\right)$,Ship Length $(L)$, Ship Width ( $B$ ),Draft ( $T$ ),Longitudinal position of center of buoyancy $\left(L_{c p}\right)$,Propeller diameter $\left(D_{P}\right)$, Disc ratio $\left(A_{e o}\right)$,Pitch ratio $\left(P_{D P}\right)$,Propeller speed $(N)$,Design speed $\left(V_{S}\right)$,Hydrofoil length $(Z C)$,Hydrofoil string length $(X C)$ ), Initial attack angle of hydrofoil ( $\alpha$ ).Using a vector representation( $X_{s p}$ ),

$$
\begin{aligned}
& X_{s p}=\left\{L_{h}, D_{1}, L_{s}, t_{s}, L, B, T,\right. \\
& \left.L_{c b}, D_{p}, A_{e o}, P_{d p}, N, V_{s}, Z C, X C, \alpha\right\}
\end{aligned}
$$

b. Manipulative design variables

Ship maneuverability is the capacity of a ship to maintain or change its state of motion. Whether a catamaran can sail straight while sailing is determined by the heading stability. In this paper, the design stability was selected as the research 
object of maneuverability. Considering comprehensively, the selected design variables are:The length of the boat $(L)$,ship beam( $B$ ),Draft( $T$ ),Block coefficient $\left(C_{b}\right)$, Water line length $\left(L_{w}\right)$,expressed in XSP, $X_{S P}=\left\{L, B, T, C_{b}, L_{w}\right\}$

c. Seakeeping design variables

Ship seakeeping is a key performance in ship navigation. In order to assess the seakeeping performance of catamaran, the six-degree-of-freedom motion response in waves is usually chosen. Because of good seakeeping performance of catamaran, while the inherent period of rolling in waves is small, the inherent period of rolling was chosen as the objective function in this paper. Selection of design variables:The length of the boat( $L$ ),ship beam( $B$ ),Draft( $T$ ),Block coefficient $\left(C_{b}\right.$ ), Water surface factor $\left(C_{w}\right)$, Catamaran $\operatorname{spacing}\left(C_{0}\right)$, Center of gravity height $\left(Z_{g}\right)$. Expressed in vector representation:

$$
X_{S P}=\left\{L, B, T, C_{b}, C_{w}, c 0, Z_{s},\right\}
$$

\section{$B$. Objective function of ship type performance optimizatio} $n$

\section{a. Rapidity objective function}

The rapidity of ships mainly consists of two aspects: the resistance to hull sailing and the efficiency of ship propulsion. The resistance is highly correlated with the displacement of the ship. In this paper, the optimization of rapidity was selected as the objective function of the resistance under the unit drainage volume:

$$
f_{1}(x)=R_{\text {total }} / \nabla
$$

In this formula, $R_{\text {total }}$ :Total resistance for navigation $(\mathrm{N}) ; \nabla$ :Drainage volume $\left(\mathrm{m}^{3}\right)$

Hull resistance consists of wave making resistance and viscous resistance.

$$
\begin{aligned}
& R_{\text {total }}=R_{W}+R_{V} \\
& =0.5 \rho V^{2} S\left(C_{W}+C_{V}\right)
\end{aligned}
$$

Among them, the wet surface area of the hull can be esti mated by the followi-ng formula:

$$
\begin{aligned}
& S=2 \times\left(2.025 L_{s}\left(T-D_{1}\right)\right. \\
& \left.+1.253 \alpha D_{1} L_{h} C_{P}^{0.5}\right)
\end{aligned}
$$

In the formula, $L_{h}{ }^{---}$Floating body length $(m) ; L_{s}---$ Prop length $(m) ; D_{1}$--- Floating body diameter $(m) ; C_{p}$ Diamond coefficient of main body; $\alpha$--- $\mathrm{A}$ coefficient, $\alpha=3.11+2.095 C_{p}$.

The viscous resistance of SWATH-USV can be divided into three parts.

$$
R_{V 1}=R_{V S}+R_{V B}+R_{A R}
$$

In the formula, $R_{V S}{ }^{---}$Pillar viscous resistance; $R_{V B}{ }^{---}$ Viscous drag of submerged body; $R_{A R}$--- Correlation resistance.

Where $R_{V S}, R_{V B}, R_{A R}$ can be estimated as:

$$
\begin{aligned}
& R_{V S}=0.5 \rho V^{2} S_{F S} C_{F S}\left(1+K_{S}\right) \\
& R_{V B}=0.5 \rho V^{2} S_{F B} C_{F B}\left(1+K_{B}\right)
\end{aligned}
$$

In the formula, where $S_{F S}$ and $S_{F B}$ mean the wet surface area of the mainstay and the main body respectively, $C_{F S}$ and $C_{F B}$ mean the wet surface area of the mainstay and the main body respectively, $1+K_{S}$ and $1+K_{B}$ mean the shape factor of the pillar and the submerged body respectively.

The eighth ITTC formula shows that:

$$
\begin{gathered}
C_{F S}=\frac{0.075}{\left(\log R_{n s}-2\right)^{2}}, R_{n s}=\frac{V L_{S}}{v} \\
C_{F B}=\frac{0.075}{\left(\log R_{n b}-2\right)^{2}}, R_{n b}=\frac{V L_{B}}{v}
\end{gathered}
$$

In the formula, where $R_{n s}$ and $R_{n b}$ mean Reynolds numbers for pillars and submerged bodies, respectively, $L_{S}$ and $L_{B}$ mean lengths of pillars and submerged bodies, $v$ means Viscosity coefficient for fluid motion.

The shape factor can be determined by the lower form:

$$
\begin{aligned}
1+K_{s}=1+\frac{t_{s}}{L_{S}}+30 \times\left(\frac{t_{s}}{L_{S}}\right)^{4} \\
1+K_{B}=1+1.5 \times\left(\frac{D}{L_{B}}\right)^{1.5}+7 \times\left(\frac{D}{L_{B}}\right)^{3}
\end{aligned}
$$

In the formula, $t_{s}$--- Maximum thickness of pillar; $D$--- The largest diameter or equivalent diameter of the body.

The relevant resistance can be calculated according to the following formula:

$$
R_{A R}=0.5 \rho V^{2}\left(S_{F S}+S_{F B}\right) \times \Delta C_{A R}
$$

In the formula, $\Delta C_{A R}=0.5 \times 10^{-3}$

According to this document [4], The wave resistance coefficient can be expressed by the following formula:

$$
\begin{aligned}
& C_{w}=0.82607-0.90011\left(L_{h} / D_{1}\right)+0.24768 d_{1}-0.048155 L_{s} \\
& +0.15261 t_{s}-0.20488 T_{1}+0.115\left(L_{h} / D_{1}\right)^{2}+0.12008\left(L_{h} / D_{1}\right) \times d_{1} \\
& -0.14762 d_{1}^{2}+0.4391\left(L_{h} / D_{1}\right) \times L_{s}+0.14061 d_{1} \times L_{s}-0.31462 L_{s}^{2} \\
& -0.14308\left(L_{h} / D_{1}\right) \times t_{s}-0.02 d_{1} \times t_{s}+0.067927 L_{s} \times t_{s}-0.06259 t_{s}^{2} \\
& -0.10343\left(L_{h} / D_{1}\right) \cdot T_{1}-0.088095 d_{1} \times T_{1}-0.3455 L_{s} \times T_{1} \\
& +0.02764 d_{s} \times T_{1}+0.28422 T_{1}^{2}
\end{aligned}
$$


In the formula, $L_{h}$---Floating body length( $m)$; $D_{1}$---Floating body diameter $(m) ; d_{1}$---The offset of the support relative to the center of the submerged $\operatorname{body}(m) ; L_{s}$--- Prop length $(m) ; t_{s}$--- Maximum width of pillar $(m) ; T_{1}$--- Submerged draught depth (This depth value is the actual water depth minus the maximum radius of the submerged body.)

The propulsion coefficient P.C was selected as a fast-moving part of the objective function:

$$
f_{2}(x)=P . C=\eta_{H} \eta_{R} \eta_{S} \eta_{0}
$$

In the formula: $\eta_{0}---$ Propeller open water efficiency; $\eta_{H}$--- Hull efficiency; $\eta_{R}$--- Relative rotation efficiency; $\eta_{s}{ }^{---}$Shafting efficiency

1) Propeller efficiency in open water

$$
\eta_{0}=\frac{K_{T}}{K_{Q}} \frac{V_{S}(1-\omega)}{n D_{P}}
$$

In the formula: $K_{T} \quad--$ Propeller thrust coefficient; $K_{Q^{---}}$Propeller torque coefficient; $V_{S}$---Design speed; $\omega$---Wake fraction; $n$---Propeller speed; $D_{p}$ Diameter of propeller.

2) Propeller hull efficiency

$$
\eta_{H}=\frac{1-t}{1-\omega}
$$

In the formula:t---Thrust reduction fraction; $\omega$---Wake fraction.

3) Relative rotation efficiency

For single propeller ship, the Holt LOWPER formula can be used as shown below:

$$
\begin{aligned}
& \eta_{R}=0.9922-0.05908 A_{e o} \\
& +0.07424\left(C_{p}-0.0225 L_{c b}\right)
\end{aligned}
$$

In the formula: $P / D$--- Ratio of disk surface; $C_{P}$--Prismatic coefficient; $L_{c b}$---the longitudinal center of buoyancy position before $0.5 \mathrm{~L}$ which was measured in percentage of $\mathrm{L}$.

4)Transmission efficiency of shaft system: $\eta_{s}=0.97$.

To sum up, the total objective function of rapidity is the weighted product of two sub-objective functions, which is expressed in the following formula:

$$
f(x)=f_{1}(x)^{\beta 1} \times f_{2}(x)^{\beta 2}
$$

In the formula, $\beta_{1} \beta_{2}$ mean the Weight number of $f_{1}(x), f_{2}(x)\left(\beta_{1}>0, \beta_{2}>0\right)$.

\section{b. Maneuverability objective function}

The linear stability was chosen as the objective function of the maneuverability of the catamaran with small water surface. The linear stability was reflected by the linear stability criterion coefficient.

The stability of linear motion $v, r$ is determined by the changing characteristics of state variables.

The stability of the system is directly related to the four acceleration hydrodynamic derivatives $Y_{v}^{\prime}, Y_{r}^{\prime}, N_{v}^{\prime}, N_{r}^{\prime}$ and the four velocity hydrodynamic derivatives. It can be expressed by the ship's linear stability coefficient:

$$
C=Y_{v}^{\prime} N_{r}^{\prime}-N_{v}^{\prime}\left(Y_{r}^{\prime}-m^{\prime}\right)
$$

Where $Y_{v}^{\prime}, Y_{r}^{\prime}, N_{v}^{\prime}, N_{r}^{\prime}$ mean Hydrodynamic derivatives for dimensionless velocity and $m^{\prime}$ means dimensionless hull quality:

$$
\begin{aligned}
& m^{\prime}=m /\left(0.5 \rho L^{3}\right) \\
& =\rho C_{B} L B T /\left(0.5 \rho L^{3}\right) \\
& =2 C_{B} \frac{B}{L} \frac{T}{L}
\end{aligned}
$$

The hydrodynamic derivatives of the hull can be derived from the regression formula of the linear hydrodynamic derivatives [8]:

$$
\begin{aligned}
& \left(Y_{v}\right)_{\text {hull }}=-0.5 \rho L d V\left(0.5 \pi+1.4 C_{b} B / L\right) \\
& \left(Y_{r}\right)_{\text {hull }}=0.5 \rho L^{2} d V(0.25 \pi) \\
& \left(N_{v}\right)_{\text {hull }}=-0.5 \rho L^{2} d V(0.54 \lambda) \\
& \left(N_{r}\right)_{\text {hull }}=-0.5 \rho L^{3} d V\left(0.54 \lambda-\lambda^{2}\right)
\end{aligned}
$$

Where $\lambda=2 d / L_{w}$ means aspect ratio. The above expressions are dimensionless:

$$
\begin{aligned}
& \left(Y_{v}^{\prime}\right)_{m h}=-d\left(0.5 \pi+1.4 C_{b} B / L\right) \\
& \left(Y_{r}^{\prime}\right)_{m h}=d(0.25 \pi) / L \\
& \left(N_{v}^{\prime}\right)_{m h}=-d(\lambda) / L \\
& \left(N_{r}^{\prime}\right)_{m h}=d\left(0.54 \lambda-\lambda^{2}\right) / L
\end{aligned}
$$

\section{c. Seakeeping objective function}

Seakeeping design variables: the length of $\operatorname{ship}(L)$,ship beam $(B)$,Draft $(T)$, Block coefficient $\left(C_{B}\right)$,Water surface coefficient $\left(C_{w}\right)$,Catamaran demihulls spacing $\left(C_{0}\right)$.The height of the center of gravity $\left(Z_{g}\right)$. These seven design variables were represented by a vector:

$$
X_{S P}=\left\{L, B, T, C_{b}, C_{w}, c 0, Z_{g},\right\}
$$

For catamaran, its stability is good, but its rolling period is short especially at high speed. Therefore, in this paper, the inherent cycle of ship was chosen as the objective function of seakeeping $f_{4}(x)$.

$$
f_{4}(x)=T_{\phi}=\frac{2 \pi}{w_{\phi}}=2 \pi \sqrt{\frac{I_{x x}^{\prime}}{\Delta h}}(s)
$$

Where Rolling

natural 
The sum of the inertia moment and the additional moment of inertia of the ship itself; $\Delta$--- Displacement; $h$--- The ship's initial stability high. $I_{x x}{ }^{--}$The moment of inertia of the ship itself; $J_{x x}$--- Additional moment of inertia.

The rolling period of a ship is related to the displacement of the ship, the high initial stability and the total inertia moment of the ship. The total inertia moment of the ship is the sum of the inertia moment and the additional inertia moment of the ship itself. The inertia moment of the ship itself depends on the mass distribution of the ship itself, and the additional inertia moment depends on the shape and height of the submerged underwater body. In order to solve the additional inertia moments of the small waterline catamaran, the two monoliths of the small waterline catamaran are separated. The additional inertia moments of the monolith are solved first, and then the additional inertia moments of the entire small waterline catamaran are fitted. A monolithic body of a SWATH-USV is regarded as an elliptical revolving body. According to the potential flow theory of ideal fluid [9], the formula of the additional moment of inertia for an elliptical body of revolution with the long axis and short axis is obtained.

$$
J_{l z z}=k_{z} \frac{4 \pi}{15} \rho a b^{2}\left(a^{2}+b^{2}\right)=k_{z} I_{z}
$$

Where, $\rho$--- Mass density of fluids; $m, I_{z}---$ Mass and rotational inertia moment of elliptical revolving body; $a, b$--- The long axis and short axis of the ellipsoid ; $k_{z}=f(b / a)$, the fitting formula, which was as follows according to a collection of illustrative plates obtained by Lamb [9]

$$
\begin{aligned}
& k_{z}=-10.539(b / a)^{6}+ \\
& 32.259(b / a)^{5} \\
& -36.216(b / a)^{4} \\
& +17.624(b / a)^{3}- \\
& 2.0814(b / a)^{2} \\
& -2.0641(b / a)+1.0
\end{aligned}
$$

where, $a=L_{h} / 2, b=D_{1} / 2$

Where, $L_{h}$---Length of floating body; $D_{1}$---Diameter of floating body.

Therefore, the added mass and additional moment of inertia of the swath can be obtained:

$$
\begin{aligned}
& J_{z z}=2\left(m_{l x}\left(1+\frac{3}{16}\left(\frac{0.5 b_{1}}{c}\right)^{3}\right) c^{2}\right) \\
& +J_{l z z}\left(1+\frac{3}{8}\left(\frac{d}{c}\right)^{3}\right)
\end{aligned}
$$

Where, $b$---Body width; $c$---The spacing between two slices; $J_{l z z}$---The additional inertia moment of a monolithic body.

The expression of initial stability of SWATH-USV

$\overline{G M}$ is expressed as:

$$
\overline{G M}=Z_{b}-Z_{g}+\overline{B M}
$$

Where, $Z_{b}$--- vertical center of buoyancy; $Z_{g}$--- Vertical coordinates of center of gravity; $\overline{B M}$--- Transverse radius of stability.

Expression of transverse core radius ( $\overline{B M}$ ) of a straight SWATH-USV ship:

$$
\begin{aligned}
& \overline{B M}=\frac{g_{1} \cdot g_{2} \cdot v}{\nabla \cdot \theta} \\
& =\frac{1}{\nabla}\left[\frac{4}{3} \int_{0}^{L} t^{3} d x+2 A_{W} b^{2}\right]
\end{aligned}
$$

Where, $t$--- Half width of design waterline $(m) ; A_{w}$---Waterplane area $\left(m^{2}\right) ; b$---Half of pillar center distance $(m)$.

\section{C . Constraint conditions for ship type performance optimiz ation}

\section{a. Equality constraint}

Equation constraints include static water buoyancy conditions, linear fitting constraints, thrust resistance balance, torque balance. The specific constraints are as follows:

(1) If the hydrostatic floatability is restricted, the ship should satisfy the positive floating state, that is, the discharge volume is equal to the gravity:

$$
\Delta=\rho L B T C_{b}
$$

(2) The thrust resistance balance constraint is equal to the total thrust of the propeller and the thrust of the hydrofoil:

$$
\begin{aligned}
& N_{p} K_{T} \rho N^{2} D_{P}^{4}(1-t)+F_{x} \\
& =R_{t}+R_{x}
\end{aligned}
$$

3) The torque balance constraint, that is, the torque supplied by the main engine to the propeller is equal to the hydrodynamic torque of the propeller:

$$
\frac{\eta_{R} \eta_{s} P_{s}}{2 \pi N}=K_{Q} \rho N^{2} D_{p}^{5}
$$

\section{b. Inequality constraint}

The propeller needs to meet the cavitation requirement according to Keller equation:

$$
\begin{aligned}
& (1.3+0.3 Z) T_{e} /\left(\left(P_{0}-P_{V}\right) D_{P}^{2}\right) \\
& +K=\left(A_{E} / A_{0}\right)_{\min }
\end{aligned}
$$

The inequality constraints are:

$$
\begin{aligned}
& (1.3+0.3 Z) T_{e} /\left(\left(P_{0}-P_{V}\right) D_{P}^{2}\right) \\
& +K-\left(A_{E} / A_{0}\right) \leq 0
\end{aligned}
$$

In the formula, $P_{0}$ means static pressure at the center of propeller shaft; $P_{V}$ means Vaporization pressure of seawater at 15 degrees Celsius; $T_{e}$ means thrust for propeller; $Z$ means numbers of blades for propeller; $K$ is a constant where Fast ship equals 0 , Other twin oars equal 0.1 and Single paddle ship equals 0.2 .

The range of the 7 design variables.

According to the specifications of sea going ships, the initial stability height, $G M>0.7 \mathrm{~m}$;

Rolling cycle: 


$$
T_{\varphi}=1.05 B / \sqrt{G M}<6.0 s
$$

\section{D . Total objective function}

The objective functions of the three subsystems of UAV, namely, rapidity, maneuverability and seakeeping, were synthesized, and the total objective functions of the synthetical optimization were constructed in the form of power exponential product as follows.

$f(x)=\frac{f_{2}(x)^{\alpha 2} \cdot f_{3}(x)^{\alpha 3} \cdot f_{4}(x)^{\alpha 4}}{f_{1}(x)^{\alpha 1}}$

Where $\alpha_{1}, \alpha_{2}, \alpha_{3}, \alpha_{4}$ are the weight of the fast, maneuverability, seakeeping of unmanned surface vehicle with the form of the product sum, $\alpha_{1} \times \alpha_{2} \times \alpha_{3} \times \alpha_{4}=1$, this paper needs comprehensive optimization of performance [10]. The larger the total objective function, the better.

\section{OPTIMIZATION CALCULATION AND ANALYSIS OFSWATH-USV}

\section{A. Introduction to optimization software}

The software consists of the following Windows: a software interface(Figure A.1) Weight setting window(FigureIII.2),Penalty factor setting window(Figure A.3)

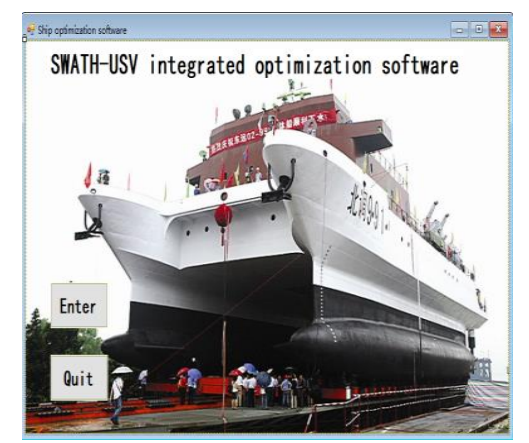

Figure A.1 The Interface of Integrated Optimization Program

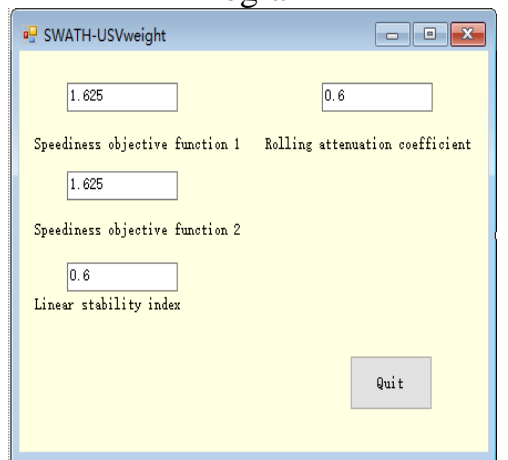

FigureIII.2 Performance Weight settings

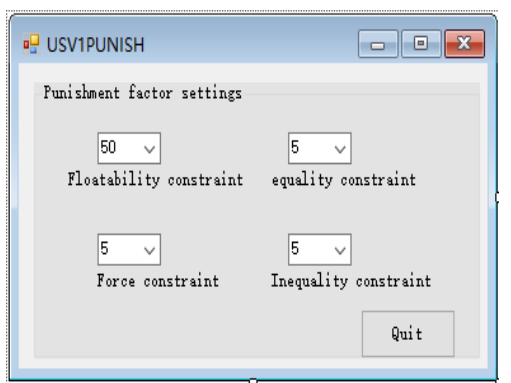

Figure A.3 Penalty factor setting window
This paper studied several optimization algorithms and algorithm improvement strategies [11] and a comprehensive optimization mathematical model of SWATH-USV was established. Using c \# language SWATH-USV comprehensive optimization program, and genetic algorithm to calculate growth mechanism, first we recorded in the calculation of the best five individual information, then according to the best of five individual information using external layered strategy, initialization, new upper and lower limits of outer carrier probability is 0.005 , we used the complex algorithm (CMP) growth mechanism of the genetic algorithm (GGA) chaos algorithm (CA) and particle swarm optimization (PSO) for a second. The genetic algorithm was combined with other hybrid algorithms for comparison.

\section{B. Different optimization algorithms are} introduced and compared

The optimized object is a piece of extension-usv, which refers to the size of the mother ship, and the displacement is set at $6.9 \mathrm{~T}$. The captain is about $8 \mathrm{~m}$, considering the basic ship shape parameters given in the last section of the mathematical model. The value range of the following design variables is given. The optimization program was carried out on the computer, and comprehensive optimization software was run. The parameters were adjusted according to the prompts. Firstly, the displacement is specified, then the upper and lower limits of design variables were set, and then an initial weight was set for each optimization subsystem. In this paper, according to the weight product of each weight and the importance of each performance given in the previous chapter, the weight of the fast objective function 1 was determined to be 1.6 , the weight of the fast objective function 2 was 1.6 , the weight of the linear stability was 0.625 , and the weight of the rolling period was 0.625 . Finally, the size of the penalty function was set and the optimization algorithm was chosen for calculation.

Genetic algorithm (GA) is a global optimization probabilistic search method with a high degree of randomness and self-adaptability. The universal genetic algorithm adopts the roulette mechanism. The main feature of the roulette mechanism is that the probability of an individual being selected to inherit the next generation is high if the fitness value is large. Such a mechanism often leads to a large number of repeated individuals (those with high fitness) in the next generation, which causes the population to lose diversity in the process of evolution. Considering the above reasons, the hierarchical strategy for genetic algorithm, the improved thought lies in the fact that the early generation of population, the worst part of the next generation of population individuals according to the previous generation of the best individual in the individual features in a nearby neighborhood randomly generated, so that we can not only ensure the population toward the optimal individual evolution, and can keep the original features of individual species to save on generation and in the growth mechanism of the genetic algorithm with varying carrier probability calculation scheme, probability in the second carrier to 0.01 , the last generation was 0.001 , algebraic calculating probability as the carrier to a linear gradient change.

Chaos Optimization Algorithm (Chaos Optimization Algorithm) is based on the ergodicity of Chaos. Chaos phenomenon is nonlinear, analogous to a random phenomenon, but there is a law within it. Because of the 
ergodicity of chaos, it is better to use chaos variable to search for optimization than random search without aim.

The basic idea of particle swarm optimization algorithm (PSO) is to find the optimal solution through the cooperation and information sharing among the individuals in the population. Meanwhile, it has the characteristics of population and evolution. Each particle represents a solution

The complex shape algorithm is an important method to solve the constrained optimization problem. The optimization process is to calculate and compare the function values of each vertex of the compound shape, and continues the mapping of points and contraction of the compound shape, so that the compound shape point is closer to the optimal solution.

In this paper, five optimization algorithms including growth mechanism genetic algorithm (GGA) GGA+GGA, GGA+ chaos algorithm (CA) GGA+ particle swarm algorithm (PSO) and GGA+ coincidence algorithm (CMP) were studied and analyzed, and the advantages and disadvantages of the algorithm were analyzed, and the optimal optimization method and optimal optimization results were obtained at last.

\section{Growth mechanism genetic algorithm} optimization calculation

The basic parameters were set as follows:

Population size:200, genetic algebra :2000-5000; Variable carrier probability :0.0001-0.001; Genetic factor 0.5 , evolutionary weight 0.5 ; No crossing, no variation, choosing growth mechanism in the calculation strategy and recording the best several values. The optimization results were shown in the following figure.

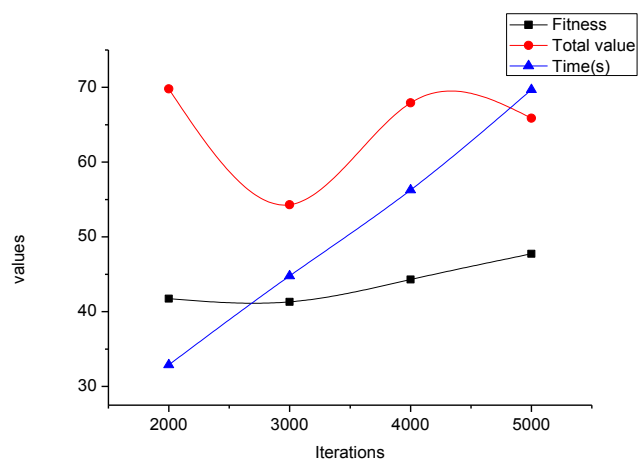

Figure C.1 The curve of Target function, Fitness, Time of iteration influenced by generations of Genetic Algorithms Accroding to

Figure C.1, as the number of iterations of genetic algorithm increases, the fitness function value shows an increasing trend and the total objective function value fluctuates slightly. As the number of iterations increases, the running time of the optimization program increases linearly. Considering the above, when the calculation number is around 4000, the overall optimization effect is the best.

\section{The hybrid algorithm optimizes the calculation}

First, we calculated the record of the best five individual information with growth mechanism of the genetic algorithm, then according to the best of five individual information adapted external layered strategy, the complex algorithm (CMP), growth mechanism of the genetic algorithm (GGA), chaos algorithm (CA) and particle swarm optimization (PSO) for a second, initialization, new upper and lower limits of outer carrier probability of 0.005 .

The following are the basic parameter settings for the different algorithms of external layering:

1) Chaos algorithm (CA): Population size: 200, calculation times:2000-5000 generations (four times), parallel number of 1 , carrier probability of 0.005

2) Particle Swarm Optimization (PSO): Population size: 200, calculation times:2000-5000 generations (four times), variable weight: $0.9-0.4$, in which the initial weight: 0.9 , the final weight: 0.4 , set the maximum speed of particle flight: 0.13

3) Complex algorithm (CMP): Population size: 200, the maximum number of iterations: 2000-5000 generations (four times), the initial mapping coefficient is 1.3 , the minimum mapping coefficient is 0.0001 , the carrier probability is 0.005

4) genetic algorithm for growth mechanism: the basic parameter setting is the same as above. Computing strategy selection: outer carrier

Table D-1 The optimization results of different mixed algorithms within 2000 iterations

\begin{tabular}{cccccc}
\hline $\begin{array}{c}\text { Computing } \\
\text { strategy }\end{array}$ & GGA & GGA+CA & GGA+PSO & GGA+GGA & GA+CMP \\
\hline $\begin{array}{c}\text { Fitness function } \\
\text { value }\end{array}$ & 41.75 & 61.19 & 58.92 & 54.23 & 62.7 \\
$\begin{array}{c}\text { Total objective } \\
\text { function }\end{array}$ & 69.79 & 67.79 & 70.9 & 67.8 & 69.34 \\
Run time /s & 32.9 & 34.94 & 65.14 & 62.83 & 49.84 \\
\hline
\end{tabular}

Table D-2 The optimization results of different mixed algorithms within 3000 iterations

\begin{tabular}{|c|c|c|c|c|c|c|}
\hline $\begin{array}{l}\text { Computing } \\
\text { Strategy }\end{array}$ & \multicolumn{2}{|c|}{ GGA } & $\mathrm{GGA}+\mathrm{CA}$ & GGA+PSO & GGA+GGA & $\mathrm{GA}+\mathrm{CMP}$ \\
\hline \multicolumn{3}{|c|}{ Fitness function } & 63.77 & 55.02 & 55.16 & 6502 \\
\hline \multicolumn{2}{|c|}{$\begin{array}{l}\text { Total objective } \\
\text { function }\end{array}$} & 54.31 & 74.67 & 71.27 & 72.24 & 75.63 \\
\hline \multicolumn{2}{|c|}{ Run time /s } & .79 & 47.99 & 88.31 & 90.33 & 59.10 \\
\hline \multicolumn{7}{|c|}{$\begin{array}{l}\text { Table D-3 The optimization results of different mixed algorithms within } \\
\qquad 4000 \text { iterations }\end{array}$} \\
\hline $\begin{array}{c}\text { Computing } \\
\text { strategy }\end{array}$ & GGA & GG & $+\mathrm{CA}$ GC & $\mathrm{GA}+\mathrm{PSO}$ & GGA+GGA & $\mathrm{GA}+\mathrm{CMP}$ \\
\hline $\begin{array}{l}\text { Fitness } \\
\text { function } \\
\text { value }\end{array}$ & 44.29 & & .12 & 62.05 & 64.24 & 68.79 \\
\hline $\begin{array}{c}\text { Total } \\
\text { objective } \\
\text { function }\end{array}$ & 67.94 & & 5.65 & 73.79 & 72.47 & 76.44 \\
\hline Run time /s & 56.27 & & .27 & 111.54 & 111.65 & 71.32 \\
\hline
\end{tabular}

Table D-4 The optimization results of different mixed algorithms within 5000 iterations

\begin{tabular}{cccccc}
\hline $\begin{array}{c}\text { Computing } \\
\text { strategy }\end{array}$ & GGA & GGA+CA & GGA+PSO & GGA+GGA & GA+CMP \\
\hline $\begin{array}{c}\text { Fitness function } \\
\text { value }\end{array}$ & 47.75 & 63.57 & 57.91 & 61.83 & 65.02 \\
$\begin{array}{c}\text { Total objective } \\
\text { function }\end{array}$ & 65.88 & 71.45 & 73.61 & 69.15 & 72.34 \\
$\begin{array}{c}\text { Run time /s } \\
\text { Run }\end{array}$ & 69.7 & 74.75 & 131.7 & 130.35 & 78.3 \\
\hline
\end{tabular}


From the results of

Table D-1, Table D-2, Table D-3,Table D-4,the hybrid algorithm optimization, the hybrid algorithm can improve the optimization effect. GGA+CA, GA+CMP can get the better fitness value. But the running time of GGA + CA is shorter, so the optimal value can be calculated by combining the genetic algorithm GGA and chaos algorithm (CA).

\section{E. Optimization results of SWATH-USV catamaran}

Through the above calculation, comprehensive fitness function value and calculation value were selected in the 4000 generation of GGA+CA algorithm of Table D-3, the optimal size of the SWATH-USV is obtained. For a small waterline catamaran with a displacement of $6.9 \mathrm{t}$, the optimal results of all design variables and the values of all sub-objective functions are shown as and respectively.

Table E-1 Design variables value of optimal results

\begin{tabular}{clccc}
\hline number & \multicolumn{1}{c}{ design variabe } & $\begin{array}{c}\text { lower } \\
\text { limit }\end{array}$ & $\begin{array}{c}\text { Upper } \\
\text { limit }\end{array}$ & $\begin{array}{c}\text { Optimal } \\
\text { value }\end{array}$ \\
\hline 1 & Submergence length $L_{h}(\mathrm{~m})$ & 7 & 7.3 & 7.152 \\
2 & Prop length $L_{S}(\mathrm{~m})$ & 6.3 & 6.6 & 6.414 \\
3 & Submersible radius $D 1(\mathrm{~m})$ & 0.7 & 0.8 & 0.753 \\
4 & Maximum width of pillar $t s$ & 0.29 & 0.35 & 0.306 \\
5 & ship' s longitude $L(\mathrm{~m})$ & 7.8 & 8.1 & 8.1 \\
6 & ship beam $B(\mathrm{~m})$ & 3.7 & 3.85 & 3.847 \\
7 & Draft $T(\mathrm{~m})$ & 0.9 & 1.15 & 0.94 \\
8 & block coefficient $C b$ & 0.2 & 0.232 & 0.23 \\
9 & lengthwise position of buoyancy & -0.1 & 0.1 & 0.016 \\
& Lcp $(\mathrm{m})$ & & & \\
10 & Propeller diameter $D p(\mathrm{~m})$ & 0.78 & 0.85 & 0.83 \\
11 & disk ratio $A_{e o}$ & 0.4 & 0.45 & 0.42 \\
12 & pitch ratio $P_{D P}$ & 0.95 & 1.05 & 1.018 \\
13 & propeller speed $N(\mathrm{rpm})$ & 270 & 350 & 294.7 \\
14 & Design speed $V_{S}(\mathrm{kn})$ & 7.5 & 8.2 & 7.554 \\
15 & demihull spacing $c O(\mathrm{~m})$ & 0.95 & 0.15 & 0.149 \\
16 & Waterline length $L w(\mathrm{~m})$ & 6.3 & 6.6 & 6.507 \\
17 & height of $\mathrm{C} . \mathrm{G} . \mathrm{Zg}(\mathrm{m})$ & 1.54 & 1.6 & 1.549 \\
18 & water plane coefficient $C w$ & 0.145 & 0.155 & 0.154 \\
19 & midship section coefficient $C m$ & 0.3 & 0.4 & 0.306 \\
20 & Hydrofoil length $Z C$ & 1.17 & 1.56 & 1.241 \\
21 & Hydrofoil string length $X C$ & 0.295 & 0.39 & 0.332 \\
22 & Hydrofoil angle of attack $a$ & 5 & 10 & 5.271 \\
\hline & & & & \\
\hline
\end{tabular}

Table E-2 The optimal results of system parameters

System total objective function and sub objective function

Fitness function value $\quad 67.126$

Total objective function value $\quad 75.17$

Speediness objective function $1 \quad 0.653$

Speediness objective function $2 \quad 0.789$

Rolling attenuation coefficient $\quad 959$

Linear stability function $\quad 0.011$

Buoyancy constraint satisfaction degree $\quad 99.98 \%$

Degree of constraint satisfaction $\quad 99.94 \%$

Torque constraint satisfaction $\quad 97.84 \%$ Penalty function value

\section{CONCLUSION}

In this paper, according to the parameters that affect the comprehensive performance of SWATH-USV, a comprehensive optimization mathematical model of SWATH-USV is established, and then the objective function of each performance is constructed as a comprehensive optimization total objective function in the form of power exponential product, and the constraints are constructed by combining design variables and actual conditions. Through external mixing of different algorithms, the merits and demerits of the combination of different algorithms are analyzed, and the best hybrid algorithm is obtained. Finally, the optimal hull forms parameters of small waterline catamaran are obtained. The following conclusions can be drawn:

1) Using single growth mechanism genetic algorithm, when the number of inheritances is about 4000, the overall optimization effect is the best;

2) Compared with a single algorithm and hybrid algorithm, the hybrid algorithm can effectively improve the fitness of the objective function;

3) Report to the four hybrid algorithms, GGA+CA and GA+CMP have better robustness and optimization ability, and the running time of $\mathrm{GGA}+\mathrm{CA}$ is shorter.

\section{REFERENCE}

[1] Ren Yaguang.Characteristics, application status and development trend of small waterline catamaran[J].Ship Design Communication, 2015,01:29-33.

[2] PECHENYUK A. Benchmarking of FlowVision software in ship flow simulation [J]. Computer Research and Simulation, 2014, 6(6): 890-9.

[3] Chen Xiaona. Study on hull form optimization of SWATH [D]; Shanghai Jiao Tong University, 2007.

[4] Xu Jie. Research and Application of Multidisciplinary Design Optimization in Small Waterline Surface Catamaran [D]; Huazhong University of Science and Technology, 2009.

[5] DEB K. Multi-objective optimization [M]. Search methodologies. Springer. 2014: 403-49.

[6] Deng Fang, Deng Weibin.Calculation of resistance performance and optimization of ship form design for catamaran[J].Journal of Qingdao University of Science and Technology (Natural Science Edition), 2015, 36 (01): 72-6.

[7] Yang Luchun, Li Xuebin, Ding Mingjun, et al. Application of multi-objective genetic algorithm and decision-making in ship type demonstration [J].Journal of Harbin Engineering University, 2012, 33 (12): 1459-64.

[8] Jia Xinle, Yang Yansheng.Mathematical model of ship motion: mechanism modeling and identification modeling[M].Dalian Maritime University Press, 1999.

[9] LAMB H. Hydrodynamics [M]. Cambridge university press, 1993.

[10] CAMPANA E, DIEZ M, LIUZZI G, et al. A multi-objective DIRECT algorithm for ship hull optimization [J]. Computational Optimization and Applications, 2018, 71(1): 53-72.

[11] Wei Zifan, Jing Shengping, Yang Songlin.Performance optimization analysis of new surface unmanned aerial vehicle based on improved genetic algorithm[J].Journal of Jiangsu University of Science and Technology (Natural Science Edition), 2017, 31 (01): 6-11. 